\title{
Development of a simple method for the analysis of phenylalanine in dried blood spot using tandem mass spectrometry
}

\author{
Amr S. Gouda and Walaa S. Nazim * (1)
}

\begin{abstract}
Background: Phenylketonuria (PKU), inborn error of metabolism, results from phenylalanine hydroxylase deficiency. PKU leads to neurological manifestations, intellectual disability, and mental disorders. Treatment depends on phenylalanine-restricted diet. Diagnosis and follow-up of PKU depends on blood phenylalanine level. The development of bacterial inhibition assay was the first routine screening test for PKU. ELISA and amino acids analyzers methods were then developed. Tandem mass spectrometry was introduced for newborn screening from dried blood spot in the late 1990s. Since then, several methods were developed, starting from using HPLC column followed by direct injection in mass spectrometer by analyte derivatization and use of external and internal standards. Kits are available for neonatal screening without derivatization using internal standards for quantitation.

Due to high PKU incidence in Egypt, it is important to continuously ameliorate the methods for neonatal diagnosis and follow-up.

Results: External standards as dried blood spots were prepared according to the previously described procedures. These standards were evaluated for phenylalanine concentration using ELISA kit. Analysis of samples was done with a singlestep elution from dried blood spot followed by 1-min mass spectrometry analysis. Validation was done according to US FDA and other related guidelines. Fifty samples were analyzed by ELISA and another 126 samples were analyzed by mass spectrometer kit. All these samples were analyzed by the developed method and no statistically significant difference was observed.
\end{abstract}

Conclusion: New simple method is developed for phenylalanine quantitation in dried blood spot using tandem mass spectrometry. This method is cost and time effective.

Keywords: Phenylalanine, Mass spectrometry, Dried blood spot, External standards

\section{Background}

Phenylketonuria (PKU) is an autosomal recessive inborn error of metabolism results from deficiency of phenylalanine hydroxylase, an enzyme that catalyzes the hydroxylation of phenylalanine to tyrosine. Accumulation of phenylalanine in blood can cross blood brain barrier leading to many detrimental effects, including profound mental retardation, seizures and autistic behavior [1]. The overall incidence of

* Correspondence: Loy_romance@yahoo.com

Biochemical Genetics Department, Human Genetics and Genome Research Division, National Research Centre, Cairo, Egypt
PKU varies widely around the world, from as high as 1 in 2600 births in Turkey [2] and 1 in 3000 births in Egypt [3] to fewer than 1 in 100,000 births in Japan [4]. Treatment depends on effectively controlling blood phenylalanine (phe) levels within therapeutic range (120 to $360 \mu \mathrm{mol} / \mathrm{L}$ ), which can be accomplished through dietary restriction of phenylalanine along with intake of a phe-free amino acid mixture (AAM), or "medical food" (MF), as the key protein source [5].

Diagnosis and follow up of PKU depends on measurement of phenylalanine in blood. The development of the

\section{Springer Open}

(C) The Author(s). 2020 Open Access This article is licensed under a Creative Commons Attribution 4.0 International License, which permits use, sharing, adaptation, distribution and reproduction in any medium or format, as long as you give appropriate credit to the original author(s) and the source, provide a link to the Creative Commons licence, and indicate if changes were made. The images or other third party material in this article are included in the article's Creative Commons licence, unless indicated otherwise in a credit line to the material. If material is not included in the article's Creative Commons licence and your intended use is not permitted by statutory regulation or exceeds the permitted use, you will need to obtain permission directly from the copyright holder. To view a copy of this licence, visit http://creativecommons.org/licenses/by/4.0/. 
first routine screening test for PKU in the early 1960s by Robert Guthrie, based on semi-quantitative measurement of Phenylalanine concentrations in blood spots by bacterial inhibition assay [6]. Colorimetric methods were developed for dried blood spots and plasma [7-9]. Later on, HPLC/UV or HPLC/fluorometry methods were developed but they require complex derivatization procedures and sophisticated sample preparation and the drawbacks of HPLC column troubleshooting [10-12]. The most frequently used method for the quantitative analysis of physiological amino acids in body fluids is ion-exchange chromatography with post-column derivatization amino acid analyzer. Although it provides excellent separation and reproducibility with minimal sample preparation, it has the disadvantage of long run time [13]. Tandem mass spectrometry was introduced for newborn screening from dried blood spot in the late 1990s [14].

Since the introduction of tandem mass spectrometry for neonatal screening, several methods were developed, either by using HPLC column like ion-pairing reversedphase liquid chromatography coupled to mass spectrometry [15] or UPLC tandem mass spectrometry [16], or by direct injection in tandem mass spectrometer by analyte derivatization and the use of both external and internal standards. Commercial kits are available for neonatal screening of amino acids and acylcarnitines with no derivatization and using internal standards for quantitation [13]. Moat et al stated that the development of ultraperformance liquid chromatography (UPLC-MS/MS), means that metabolic laboratories now have the capability to routinely analyze DBS specimens with superior specificity and sensitivity [17]

Due to the high incidence of PKU in Egypt and the large number of neonates, it is logical to continuously develop the analytical methods for neonatal screening and follow up regarding to time and cost factors, i.e., a method capable to analyze the largest number of samples with least time and cost. Accordingly, the study aimed to develop a simple method with lowest cost and time for the quantitative determination of phenylalanine in dried blood spot using tandem mass spectrometer.

\section{Methods}

\section{Preparation of external standards}

A set of external standards were prepared according to Elvers et al., 2007 [18]

Blood on heparin was obtained from a healthy donor. Hematocrit was adjusted to $50 \%$ and divided into 6 portions named A-F. These portions were enriched by phenylalanine stock solution $(0.1 \mathrm{mmol} / \mathrm{ml}$ in saline $)$ to prepare 6 different standard concentrations in the range $0-800 \mu \mathrm{mol} / \mathrm{l}$ according to Table 1 . The portions were homogenized on a roller bank for $5 \mathrm{~h}$. Fifty microliters from each standard was spotted on Schleicher \& Schuell
903 filter paper and left to dry overnight at room temperature and then the cards were packed in a dry bag with silica gel and kept at $-20{ }^{\circ} \mathrm{C}$.

Phenylalanine concentration in standard A (basal blood) was determined using ELISA kit (Quantase neonatal phenylalanine screening, BioRad). The concentration of phenylalanine in the enriched blood was based on the concentration in basal blood and the amount of phenylalanine added to the blood.

\section{Sample preparation}

Three millimeters disc was punched from dried blood spot of each standard and sample in a 96-well plate. Elution of phenylalanine was done by the addition of $100 \mu \mathrm{l}$ methanol and incubation for 20 minutes on plate shaker set at 350 $\mathrm{rpm}$. The elute was transferred to a new 96-well plate.

\section{LC/MS/MS instrumentation and conditions}

Mass spectrometer Waters Xevo TQD triple quadrupole equipped with electrospray ionization source coupled to Aquity UPLC system, Masslynx 4.1 software was used for analysis. Ten microliters of elute was injected using acetonitrile and deionized water in the ratio 1:1 as a mobile phase. The gradient flow was adjusted as follows: $0.27 \mathrm{ml} / \mathrm{min}$ for $7.5 \mathrm{~s}, 0.02 \mathrm{ml} / \mathrm{min}$ for $37.5 \mathrm{~s}, 0.6 \mathrm{ml} / \mathrm{min}$ for $6 \mathrm{~s}$, and $0.27 \mathrm{ml} / \mathrm{min}$ for $9 \mathrm{~s}$. The ion source was operated in positive mode. Multiple reaction monitoring mode was used with mass transition $165.98>120.22$, cone and collision energy were set at 18 and $12 \mathrm{~V}$, respectively. The mass transition is shown in Fig. 1, while Fig. 2 shows a chromatogram of the standard A.

\section{Method validation}

Validation was done for the lower limit of quantitation (LLOQ), linearity, accuracy, precision, and stability according to US FDA and other related guidelines (Brazilian Health Regulatory Agency and European Medicines Agency) for the validation of a bioanalytical method [19].

Calibration linearity was evaluated with the six prepared standards at six concentrations. The calibration curve was constructed by plotting the peak area of phenylalanine versus the nominal concentrations.

The LLOQ was defined as the lowest concentration that resulted in a peak with a signal-to-noise ratio greater than 10 .

Three dried blood spot samples with three different concentration of phenylalanine (low, medium, and high concentrations) were analyzed in five replicates in the same day to determine the intraday precision and accuracy. The same samples were analyzed on three successive days to determine the interday precision and accuracy. Precision was evaluated as the percentage relative standard deviation (RSD), and accuracy was defined as the deviation from nominal value (relative error, $\mathrm{RE}$ ). 
Table 1 Preparation of standards

\begin{tabular}{llllll}
\hline Standard & Blood $[\mathrm{ml}]$ & Removed plasma $(\boldsymbol{\mu l})$ & Phenylalanine stock $(\boldsymbol{\mu l})$ & Saline $(\boldsymbol{\mu l})$ & Concentration-basal concentration $(\boldsymbol{\mu m o l} / \mathbf{l})$ \\
\hline A & 20 & 580 & 0 & 580 & 0 \\
B & 20 & 580 & 10 & 570 & 50 \\
C & 20 & 580 & 20 & 560 & 100 \\
D & 20 & 580 & 45 & 535 & 225 \\
E & 20 & 580 & 80 & 500 & 400 \\
F & 20 & 580 & 160 & 420 & 800 \\
\hline
\end{tabular}

The stability was assessed with samples spiked at two levels, low and high, under a variety of conditions: 7 days at room temperature, a month at $4{ }^{\circ} \mathrm{C}$.

To compare the efficiency of this method to currently available methods, 50 samples were analyzed by both ELISA and the developed method and 126 other samples were analyzed by both the developed method and neonatal Chromsystem mass spectrometry kit for amino acids and acylcarnitines. Samples were collected from PKU patients referred for follow up or neonates referred for neonatal screening. A signed written informed consent was obtained after approval of the Medical Research Ethics Committee of the National Research Centre. Results were analyzed in GraphPad Prism 7. The Results obtained from each of the three methods of phenylalanine quantitation in dried blood spots were first tested to determine whether they follow normal distribution using the D'Agostino-Pearson omnibus normality test. Since none of the tested datasets passed the normality test, correlation between each two of them was analyzed using the nonparametric, Spearman correlation test. To assess the agreement of the new method with the established methods, Bland-Altman plot was used. Unless otherwise specified, the results of the statistics were reported with the corresponding 95\% CI and P values.

\section{Results}

The phenylalanine concentration in the basal blood measured by ELISA from dried blood spot was determined to be $0.94 \mathrm{mg} / \mathrm{dl}(56.9 \mu \mathrm{mol} / \mathrm{l})$. The concentrations of the other prepared standards were determined by addition of the added amount to the basal blood concentration.

\begin{tabular}{lllllll}
\hline Standard & A & B & C & D & E & F \\
Concentration & 0.94 & 1.76 & 2.59 & 4.66 & 7.54 & 14.15 \\
mg/dl ( $\boldsymbol{\mu m o l} /$ & $(56.9)$ & $(106.9)$ & $(156.9)$ & $(281.9)$ & $(456.9)$ & $(856.9)$ \\
l) & & & & & & \\
\hline
\end{tabular}

Analysis of the standards set with the developed method was done and a calibration curve was constructed and shown in Fig. 3.

LLOQ was determined equals to $0.1 \mathrm{mg} / \mathrm{dl}$ with a peak signal to noise ratio greater than 10 .

The 3 samples (low, medium and high concentrations) were analyzed for intraday and interday precision and accuracy. The RSD of intraday precision was $7.5 \%, 6.9 \%$, and $18.6 \%$ while the RSD for interday precision was $12.5 \%, 7.2 \%$, and $15.4 \%$ for low, medium, and high concentration samples, respectively. The RE of intraday accuracy was $8.1 \%, 14.5 \%$, and $18.1 \%$, while that of interday accuracy was $3.2 \%,-2.9 \%$, and $-12.8 \%$ for low, medium, and high concentration samples, respectively.

After three freeze-thaw cycles, the concentrations of phenylalanine in dried blood spot deviated by less than $\pm 15 \%$ from their nominal concentrations $(4.7 \%$ for low concentration and $5.9 \%$ for high concentration). The QC samples at low and high levels were stable for up to 7 days at room temperature, and there were no significant

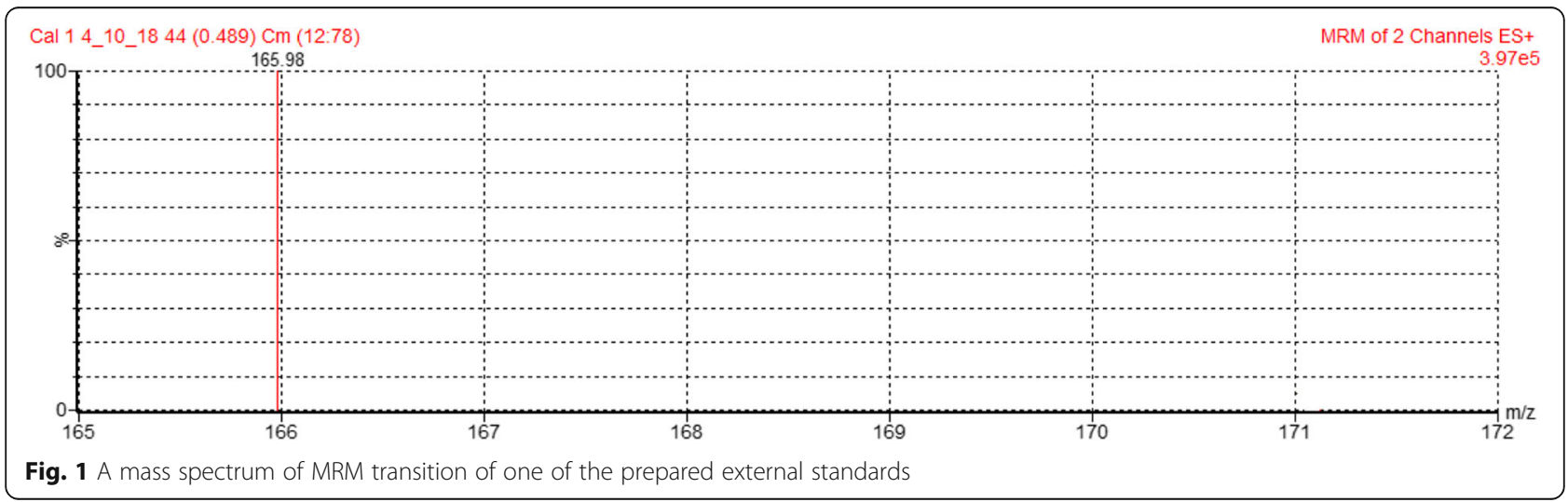




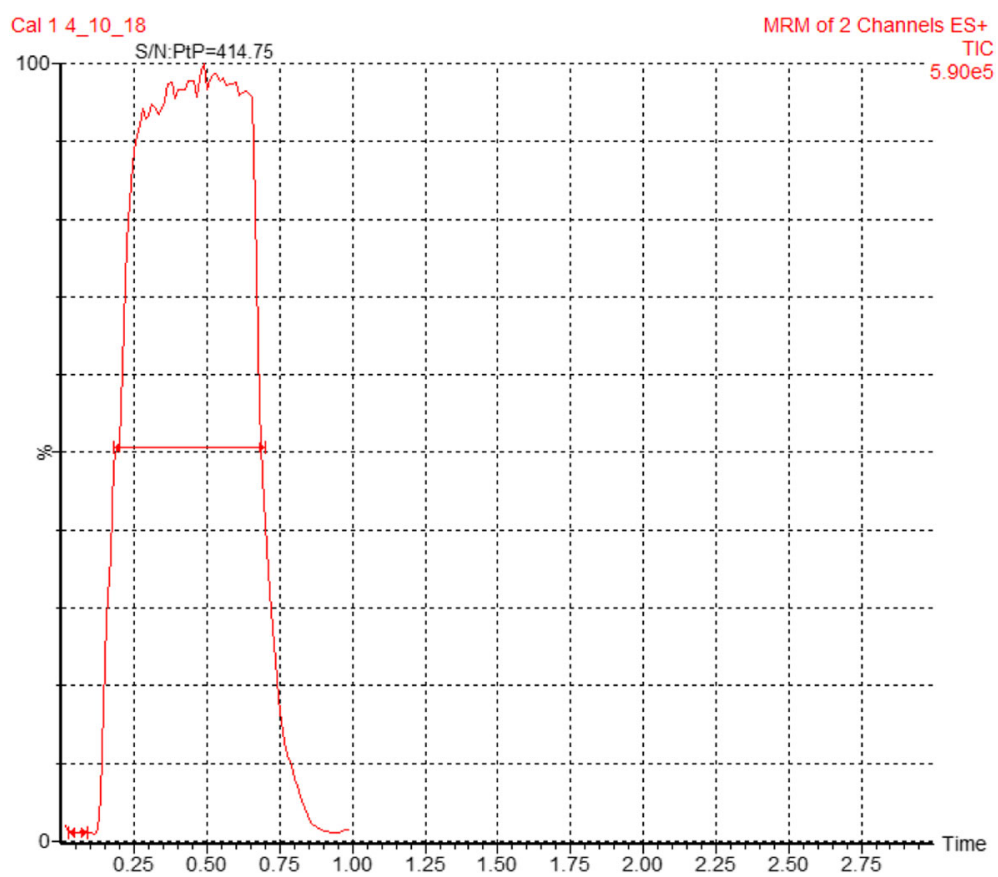

Fig. 2 A chromatogram of the calibrator A showing the signal to noise ratio $(S / N)$

differences in the responses after one month at $4{ }^{\circ} \mathrm{C}$ with RE less than $\pm 15 \%$.

Statistical analysis revealed a statistically significant correlation between results of the 50 samples analyzed by ELISA and the developed method (Spearman correlation $(r)=0.76, \mathrm{CI}=0.59-0.87, P<0.0001)$. Also a statistically significant correlation was detected between results of the 126 samples analyzed by neonatal Chromsystem for amino acids and acylcarnitines mass spectrometry kit and the developed method (Spearman correlation $(r)=0.63$,

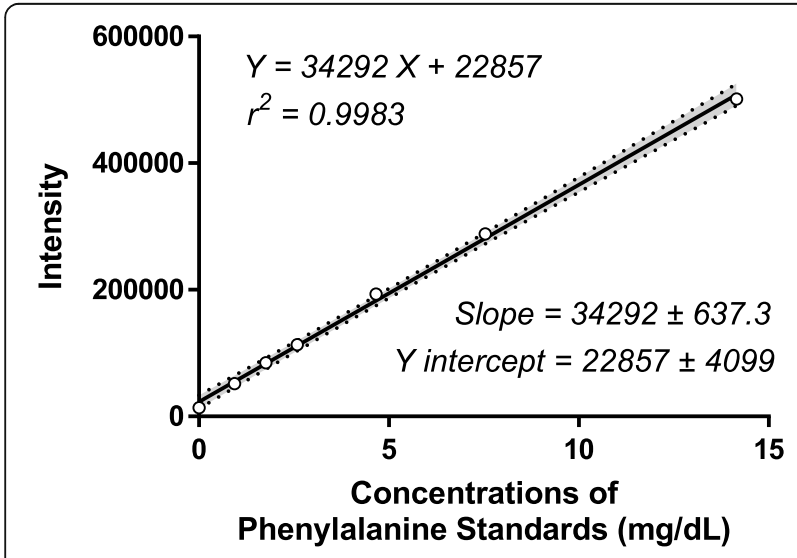

Fig. 3 Calibration curve for estimating phenylalanine concentration in dried blood spots. The shaded area between the lower and upper dotted lines represents the $95 \% \mathrm{Cl}$ of the curve, showing the equation and coefficient of determination $\left(r^{2}\right)$. Slope and $Y$ intercept are reported as mean $\pm \mathrm{SE}$
$\mathrm{CI}=0.51-0.72, P<0.0001)$. The agreement between the developed method and ELISA or neonatal was further investigated using Bland-Altman plots (Figs. 4 and 5).

\section{Discussion}

\section{Method development}

The multiple reaction monitoring (MRM) transitions as well as parameters, including collision energy, cone, and capillary voltage were optimized by direct infusion to obtain the highest sensitivity.

Elution was done using several eluents (deionized water, acetonitrile, methanol, water and acetonitrile, water and methanol) the best one that gives the highest sensitivity was methanol. Water caused sample hemolysis which increased the matrix effect and decreased sensitivity.

Due to the high selectivity of tandem mass spectrometry, the application did not require the use of an HPLC column. This enables a short run time $(1 \mathrm{~min})$ and avoids the usual troubleshooting of HPLC columns. Gradient flow rate was used to increase sensitivity.

Elution of amino acids and acylcarnitines was done by methanol in previous studies. Applications of these studies also did not include column, although derivatization of amino acids was done $[20,21]$. The derivatization step that is excluded in this method consumes more effort, time, and chemicals.

To avoid ion suppression effect and accurate determination of phenylalanine, the external standards were prepared in the form of dried blood spot to be the same matrix effect and recovery. 


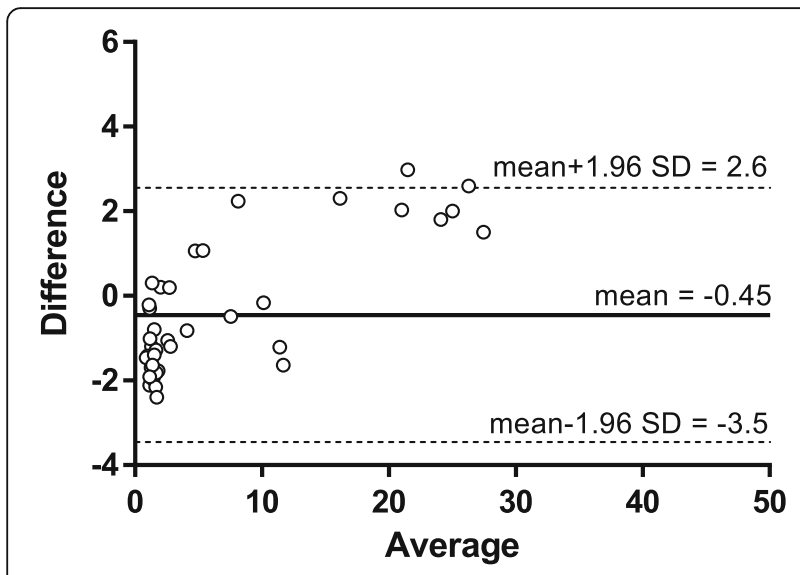

Fig. 4 Bland-Altman plot of the difference between the new method and ELISA vs. the average of the two methods. The continuous line represents the mean of the differences between the two methods while the dotted lines represent the limits of agreement from $-1.96 \mathrm{SD}$ to $+1.96 \mathrm{SD}$

\section{Method validation}

According to linearity, the calibration curve was linear with calibration regression coefficient $(r)$ equals to 0.999 . Response was accurate at all points with RE ranges from -10.5 to 6.4 .

From the results of the stability tests, it can be concluded that the developed method is reliable and robust within the analytical range.

The intraday and interday precision and accuracy of the samples at the three concentration levels were within the acceptable range, concluding the developed method to be accurate and precise.

As mentioned, the developed method did not use internal standard, and due to the difficulty in obtaining a

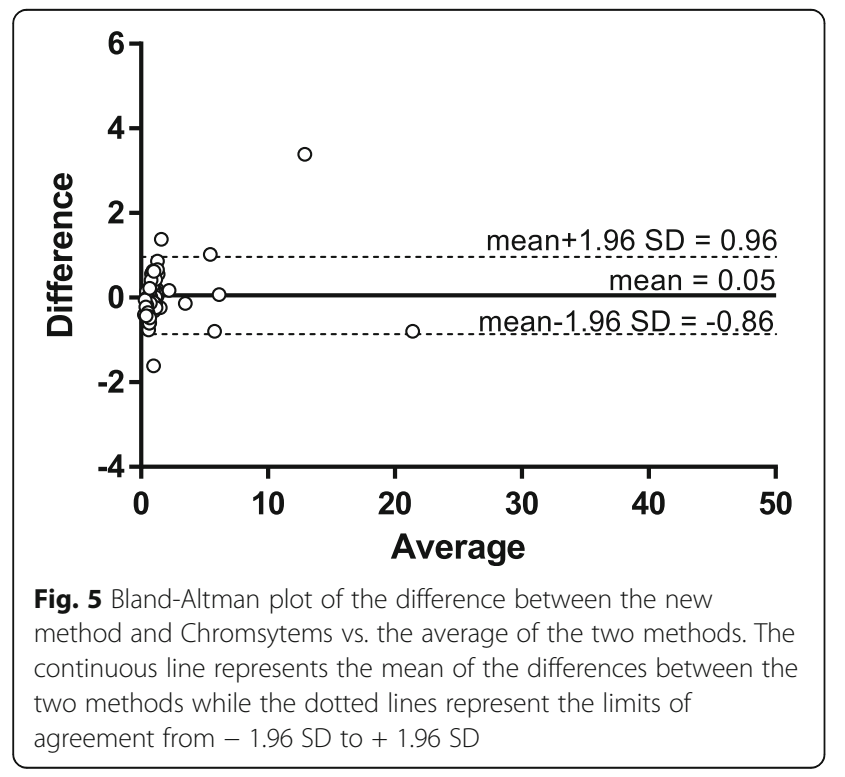

blood free from phenylalanine to be used as a blank sample, it was not possible to perform the recovery and matrix effect experiments [22]. This can be overcome by comparison of the results obtained from this method to previously established methods that are used in diagnosis.

The results were compared to both ELISA technique using (Quantase neonatal phenylalanine screening, Biorad) and neonatal Chromsystem for amino acids and acylcarnitines mass spectrometry kit. Moderate to strong correlation was detected with Spearman correlation coefficient equals to 0.76 and 0.63 for the developed method compared to ELISA and neonatal mass spectrometry kit respectively. Comparison of phenylalanine values measured by the developed method to both ELISA and neonatal methods demonstrated agreement. However, when (ELISA + New Method)/2 is larger than 16 $\mathrm{mg} / \mathrm{dl}$, New Method is always higher than ELISA due to the upper limit of quantitation by the ELISA kit is $16 \mathrm{mg} / \mathrm{dl}$. This means that the results are reliable and can be used for diagnosis and follow-up of phenylketonuria.

\section{Conclusions}

In conclusion, a simple method is developed for phenylalanine quantitation in dried blood spot using tandem mass spectrometry. This method is cost effective; no need for the expensive stable isotope internal standard, and no HPLC column is used. It is also time effective; simple one step sample elution without any derivatization and 1-min whole run time on mass spectrometry.

\section{Abbreviations}

LLOQ: Lower limit of quantitation; MRM: Multiple reaction monitoring; Phe: Phenylalanine; PKU: Phenyl ketonuria; RE: Relative error; RSD: Relative standard deviation

\section{Acknowlegements}

Not applicable

\section{Authors' contributions}

Both authors contributed to the selection of the study point and study design. Material preparation was done by both authors; data collection and analysis were done by WN author. The first draft was written by WN author, while it was revised by AG author. Both authors read and approved the final manuscript.

\section{Funding}

No funding

\section{Availability of data and materials Not applicable}

\section{Ethics approval and consent to participate}

Approval was obtained from the medical research ethical committee at the National Research Centre (reference no. FWA 00014747). The procedures used in this study adhere to the tenets of the Declaration of Helsinki. A signed written informed consent was obtained from all individual participants included in the study or their legal guardians.

\section{Consent for publication} Not applicable 


\section{Competing interests}

The authors declare that they have no competing interests.

Received: 2 July 2020 Accepted: 17 September 2020

Published online: 04 December 2020

\section{References}

1. Hood A, Grange DK, Christ SE, Steiner R, White DA (2014) Variability in phenylalanine control predicts IQ and executive abilities in children with phenylketonuria. Mol Genet Metab. 111(4):445-451

2. Ozalp I, Coskun T, Tokatli A, Kalkanoglu HS, Dursun A, Tokol S et al (2001) Newborn PKU screening in Turkey: at present and organization for future. Turk J Pediatr. 43(2):97-101

3. Araby HE, Fateen E, Gouda A (2009) Screening for phenylketonuria and galactosemia among Egyptian newborns in Menoufiya governorate. Egyptian J Med Human Genet 10:2

4. Aoki K, Ohwada M, Kitagawa T (2007) Long-term follow-up study of patients with phenylketonuria detected by the newborn screening programme in Japan. J Inherit Metab Dis. 30(4):608

5. Vockley J, Andersson HC, Antshel KM, Braverman NE, Burton BK, Frazier DM et al (2014) Phenylalanine hydroxylase deficiency: diagnosis and management quideline. Genet Med. 16(2):188-200

6. Guthrie R, Susi A (1963) A Simple Phenylalanine Method for Detecting Phenylketonuria in Large Populations of Newborn Infants. Pediatrics. 32: 338-343

7. Wendel U, Hummel W, Langenbeck U (1989) Monitoring of phenylketonuria: a colorimetric method for the determination of plasma phenylalanine using L-phenylalanine dehydrogenase. Anal Biochem. 180(1): 91-94

8. Randell EW, Lehotay DC (1996) An automated enzymatic method on the Roche COBAS MIRA S for monitoring phenylalanine in dried blood spots of patients with phenylketonuria. Clin Biochem. 29(2):133-138

9. Wibrand $F$ (2004) A microplate-based enzymatic assay for the simultaneous determination of phenylalanine and tyrosine in serum. Clin Chim Acta. 347(1-2):89-96

10. Mo XM, Li Y, Tang AG, Ren YP (2013) Simultaneous determination of phenylalanine and tyrosine in peripheral capillary blood by HPLC with ultraviolet detection. Clin Biochem. 46(12):1074-1078

11. Kand'ar R, Zakova P (2009) Determination of phenylalanine and tyrosine in plasma and dried blood samples using HPLC with fluorescence detection. J Chromatogr B Analyt Technol Biomed Life Sci. 877(30):3926-3929

12. Pecce R, Scolamiero E, Ingenito L, Parenti G, Ruoppolo M (2013) Optimization of an HPLC method for phenylalanine and tyrosine quantization in dried blood spot. Clin Biochem. 46(18):1892-1895

13. Groselj U, Murko S, Zerjav Tansek M, Kovac J, Trampus Bakija A, Repic Lampret B et al (2015) Comparison of tandem mass spectrometry and amino acid analyzer for phenylalanine and tyrosine monitoring--implications for clinical management of patients with hyperphenylalaninemia. Clin Biochem. 48(1-2):14-18

14. Chace DH, Millington DS, Terada N, Kahler SG, Roe CR, Hofman LF (1993) Rapid diagnosis of phenylketonuria by quantitative analysis for phenylalanine and tyrosine in neonatal blood spots by tandem mass spectrometry. Clin Chem. 39(1):66-71

15. Piraud M, Vianey-Saban C, Petritis K, Elfakir C, Steghens JP, Bouchu D (2005) Ion-pairing reversed-phase liquid chromatography/electrospray ionization mass spectrometric analysis of 76 underivatized amino acids of biological interest: a new tool for the diagnosis of inherited disorders of amino acid metabolism. Rapid Commun Mass Spectrom. 19(12):1587-1602

16. Galba J, Michalicova A, Parrak V, Novak M, Kovac A (2016) Quantitative analysis of phenylalanine, tyrosine, tryptophan and kynurenine in rat model for tauopathies by ultra-high performance liquid chromatography with fluorescence and mass spectrometry detection. J Pharm Biomed Anal. 117: 85-90

17. Moat SJ, George SR, Carling SR (2020) Use of dried blood spot specimens to monitor patients with inherited metabolic disorders. Int. J. Neonatal Screening. 6(26):1-17

18. Elvers LH, Loeber JG, Dhondt JL, Fukushi M, Hannon WH, Torresani T et al (2007) First ISNS Reference Preparation for Neonatal Screening for thyrotropin, phenylalanine and 17alpha-hydroxyprogesterone in blood spots. J Inherit Metab Dis. 30(4):609
19. Kollipara S, Bende G, Agarwal N, Varshney B, Paliwal J (2011) International Guidelines for Bioanalytical Method Validation: A Comparison and Discussion on Current Scenario. chromatographia 73(3-4):201-217

20. Cavedon CT, Bourdoux P, Mertens K, Van Thi HV, Herremans N, de Laet C et al (2005) Age-related variations in acylcarnitine and free carnitine concentrations measured by tandem mass spectrometry. Clin Chem. 51(4): 745-752

21. Schulze A, Lindner M, Kohlmuller D, Olgemoller K, Mayatepek E, Hoffmann GF (2003) Expanded newborn screening for inborn errors of metabolism by electrospray ionization-tandem mass spectrometry: results, outcome, and implications. Pediatrics. 111(6 Pt 1):1399-1406

22. Zhou W, Yang S, Wang PG (2017) Matrix effects and application of matrix effect factor. Bioanalysis. 9(23):1839-1844

\section{Publisher's Note}

Springer Nature remains neutral with regard to jurisdictional claims in published maps and institutional affiliations.

\section{Submit your manuscript to a SpringerOpen ${ }^{\circ}$ journal and benefit from:}

- Convenient online submission

- Rigorous peer review

- Open access: articles freely available online

High visibility within the field

- Retaining the copyright to your article

Submit your next manuscript at $\boldsymbol{\nabla}$ springeropen.com 